\title{
The Impact of Serum Amyloid P-Component on Gene Expression in RAW264.7 Mouse Macrophages
}

\author{
Dan Xi, Jinzhen Zhao, Jichen Liu, Haowei Xiong, Wenshuai He, Jing Hu, \\ Wenyan Lai, and Zhigang Guo \\ Division of Cardiology, Huiqiao Medical Center, Nanfang Hospital, Southern Medical University, Guangzhou, \\ Guangdong 510515, China \\ Correspondence should be addressed to Wenyan Lai; frame007@163.com and Zhigang Guo; guozhigang126@126.com
}

Received 29 January 2016; Revised 3 April 2016; Accepted 10 April 2016

Academic Editor: Gernot Zissel

Copyright (C) 2016 Dan Xi et al. This is an open access article distributed under the Creative Commons Attribution License, which permits unrestricted use, distribution, and reproduction in any medium, provided the original work is properly cited.

Serum amyloid P-component (SAP) contributes to host defense and prevents fibrosis. Macrophages are the most abundant inflammatory cell type in atherosclerotic plaques. In the present study, using ${ }^{3} \mathrm{H}$-cholesterol-labeled counting radioactivity assay, we demonstrated that the apoAI-mediated cholesterol efflux in RAW264.7 macrophages was increased by SAP treatment in a time- and dose-dependent manner. We analyzed global gene expression changes upon SAP treatment using RNA sequencing. As a result, a total of 175 differentially expressed genes were identified, of which 134 genes were downregulated and 41 genes were upregulated in SAP treated cells compared to control cells. Quantitative RT-PCR analysis confirmed decreased expression of 5 genes and an increase in expression of 1 gene upon SAP treatment. Gene ontology analysis showed that genes involved in response to stimulus were significantly enriched in differentially expressed genes. Beyond protein-coding genes, we also identified 8 differentially expressed long noncoding RNAs. Our study may provide new insights into mechanisms underlying the functional role of SAP in macrophages.

\section{Introduction}

Serum amyloid P-component (SAP) is a member of the pentraxin protein family which was first isolated and identified in "amyloid" pathological deposits. Under normal conditions, SAP is thought to be synthesized and secreted only in hepatocytes. In some diseases, SAP can also be generated by macrophages and smooth muscle cells such as in the atherosclerotic aortic intima [1]. In humans, SAP is constitutively expressed and contributes to host defense through the classical pathway. Studies indicate that SAP does not exist in normal aortic intima but deposits in human atherosclerotic aortic intima and that plasma SAP levels are positively associated with cardiovascular disease [2]. Additionally, SAP binds to amyloid-like structures in oxidized low density lipoprotein (ox-LDL) and prevents lipid uptake by macrophages, suggesting an important role for SAP in atherosclerosis [3]. It will be necessary to further explore the roles of SAP in lipid metabolism and atherosclerosis.

Atherosclerosis has been known as an inflammatory disease for many years. Macrophages are an essential component of the innate immunity and mediate inflammatory responses by recognizing pathogens and producing proinflammatory mediators. Macrophages are the most abundant inflammatory cell type in atherosclerotic plaques. Macrophages are transformed into foam cells upon modified low density lipoprotein uptake and their subsequent death within lesions fuels the formation of the highly proinflammatory and thrombogenic lipid-rich necrotic core $[4,5]$. A study revealed that SAP may participate in cholesterol removal from macrophages through its role in promoting cholesterol efflux [6]. The murine macrophage cell line RAW264.7 is easy to propagate and possesses high efficiency for DNA transfection and sensitivity to RNA interference. This cell line is often used in vitro to evaluate the effects of inflammation process [7] in progress of atherosclerosis [8] and especially in cholesterol efflux research [9]. It is a suitable cell line for experiments and our research group has done many experiments using this cell line $[10,11]$.

By decreasing the numbers of fibrocytes and profibrotic macrophages [12], exogenous administration of SAP has been shown to reduce fibrosis in animal models $[13,14]$. 
Recently, it has also been demonstrated that a type of recombinant human SAP (PRM-151) is able to reduce fibrocytes in pulmonary fibrosis patients [15]. The decreased accumulation of fibrocytes by SAP might be due to reduced leukocyte recruitment via lowering the levels of inflammatory cytokines [16]. Our research group has found that SAP levels significantly increased in acute coronary syndrome (ACS) patients compared with controls [17]. Furthermore, we also revealed that HDL subfractions from ACS patients possess significantly elevated SAP levels, suggesting that SAP may have vital effects on HDL subfraction functions [17]. In the present study, we investigated the effect of SAP on cholesterol efflux in macrophages, and we also attempted to analyze global gene changes associated with RAW264.7 macrophage cells after SAP treatment using RNA sequencing. Our data afforded the opportunity to test the hypothesis that SAP exerts global transcriptional effects on macrophages.

\section{Materials and Methods}

2.1. Cell Culture and Treatment. Murine RAW264.7 macrophage cell line was purchased from China Center for Type Culture Collection (CCTCC, Wuhan, China). The RAW264.7 macrophages were seeded in six-well flat bottom culture at $1.0 \times 10^{6}$ cells per well in DMEM (Gibco, Life Technologies, China) containing 10\% fetal bovine serum (Gibco, Life Technologies, EU Approved Origin, South America) and maintained at $37^{\circ} \mathrm{C}$ in a humidified atmosphere of $5 \% \mathrm{CO}_{2}$. Human serum amyloid P-component (SAP) was purchased from Calbiochem (Calbiochem, EMD Chemicals, MA, USA). SAP was frozen in PBS without the sodium azide preservative. Before experiment, cells were synchronized by changing DMEM supplemented with $2 \%$ bovine serum albumin (BSA, Amresco, USA) for $24 \mathrm{~h}$. Then, cells were cultured in primary six-well plates and treated with different concentrations of SAP. BSA served as control.

2.2. Assay of apoAI-Mediated Cholesterol Efflux. Murine RAW264.7 macrophage cells were incubated in culture medium containing $30 \mu \mathrm{g} / \mathrm{mL}$ ox-LDL (Yiyuan Biotechnologies, Guangzhou, China) and $1 \mu \mathrm{Ci} / \mathrm{mL}\left[1 \alpha, 2 \alpha-{ }^{3} \mathrm{H}\right]-$ cholesterol (Amersham Life Science, USA) for $24 \mathrm{~h}$. After being washed with serum-free medium, the cells were incubated in DMEM with $0.2 \%$ BSA containing various concentrations of SAP $(0 \sim 10 \mu \mathrm{M}, 1 \mu \mathrm{M}$ SAP $=127 \mathrm{mg} / \mathrm{mL}$ of SAP pentamers) for another $6 \mathrm{~h}, 24 \mathrm{~h}, 48 \mathrm{~h}$, respectively. Cells were subsequently incubated in serum-free medium (without BSA) with or without $10 \mu \mathrm{g} / \mathrm{mL}$ apoAI (Calbiochem, Germany) for $6 \mathrm{~h}$. Then the incubation medium was collected while the cells were washed with PBS and lysed with $0.1 \mathrm{M} \mathrm{NaOH}$. Lastly, the radioactivity of medium and cell lysates was measured by liquid scintillation spectrometry. The cholesterol efflux rate was presented as the ${ }^{3} \mathrm{H}$-cholesterol radioactivity of medium normalized to total ${ }^{3} \mathrm{H}$-cholesterol radioactivity [18].

2.3. RNA Sequencing. Before RNA isolation, cells were washed by PBS for three times. Total RNA was isolated using TRIzol reagent (Invitrogen, USA). Samples were sent to Beijing Genomics Institute (BGI) for further bioinformatic analysis. BGI is a genome sequencing center headquartered in Shenzhen, Guangdong Province, China. RNA purity was assessed using the ND-1000 NanoDrop. The A260/A280 ratio for each RNA sample was greater than 1.8 and the $A 260 / A 230$ ratio was greater than 2.0. RNA integrity was evaluated using the Agilent 2100 TapeStation. Only RNA samples with a RINe value above 7.0 were retained. Oligo(dT) magnetic beads were used to isolate poly $(\mathrm{A})+$ mRNAs. The mRNAs were fragmented to approximately $200 \mathrm{bp}$ in fragmentation buffer. Subsequently, these fragments were used as templates for first-strand and second-strand cDNA synthesis. The double-stranded cDNA fragments were purified, end-repaired, and then ligated to sequencing adapters. After purification, suitable fragments were enriched by PCR amplification according to instructions of TruSeq ${ }^{\circledR}$ RNA LT/HT Sample Prep Kit (Illumina). Finally, PCR products were purified and quantified for high-throughput sequencing using the Illumina $\mathrm{HiSeq}^{\mathrm{TM}} 2500$.

2.4. Bioinformatic Analysis of RNA Sequencing Data. A computational pipeline was employed to process the raw data from RNA sequencing. Sequence data in fastq format were filtered to remove reads with unknown nucleotides. Clean reads were mapped to mouse reference genome $\mathrm{mm} 9$ by using Tophat v1.4.0 [19]. No more than two mismatches were allowed. The mapped reads were assembled into genes and transcripts by Cufflinks v1.3.0 [20]. Gene models were downloaded from the UCSC RefSeq annotation. Gene expression levels were calculated using fragments per kilobase of transcript per million mapped fragments (FPKM) in Cufflinks. Differentially expressed genes were chosen according to the criteria of fold change $>2$ and FDR $<0.05$. GO enrichment analysis was performed by using BiNGO 2.3 with the GOslim dataset [21]. To test for enrichment, a hypergeometric test was conducted followed by Benjamini and Hochberg multiple test correction. The adjusted $p$ value $<0.05$ was used as the significance threshold to identify enriched categories. The STRING database v10.0 (http://string-db.org/) was used to create gene network. Connectivity for each gene was analyzed by in-house MATLAB scripts. The connectivity threshold value for hub genes was the mean plus two standard deviations.

2.5. Validation by Quantitative RT-PCR. The total RNA was extracted with RNAiso Plus (TaKaRa Biotechnology, Dalian, China). Reverse transcription was performed at $37^{\circ} \mathrm{C}$ for $15 \mathrm{~min}$ followed by $98^{\circ} \mathrm{C}$ for $5 \mathrm{~min}$ using ReverTra Ace qPCR RT Kit (Toyobo, Osaka, Japan). Quantitative PCR was performed using THUNDERBIRD SYBR qPCR Mix (Toyobo, Osaka, Japan) on the Applied Biosystems 7500 (Life Technologies). The program was as follows: $95^{\circ} \mathrm{C}$ for $60 \mathrm{sec}$, followed by 40 cycles of $95^{\circ} \mathrm{C}$ for $15 \mathrm{sec}$ and $60^{\circ} \mathrm{C}$ for $45 \mathrm{sec}$. All reactions were run in triplicate. The GAPDH gene was amplified as a reference gene for normalization. Data were analyzed using $2^{-\Delta \Delta \mathrm{Ct}}$ method. Primers used in this study 


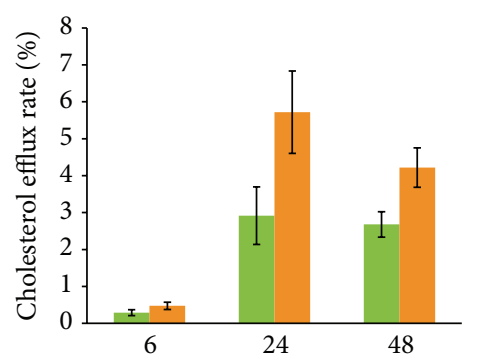

(h)

- Control

- SAP $(10 \mu \mathrm{g} / \mathrm{mL})$

(a)

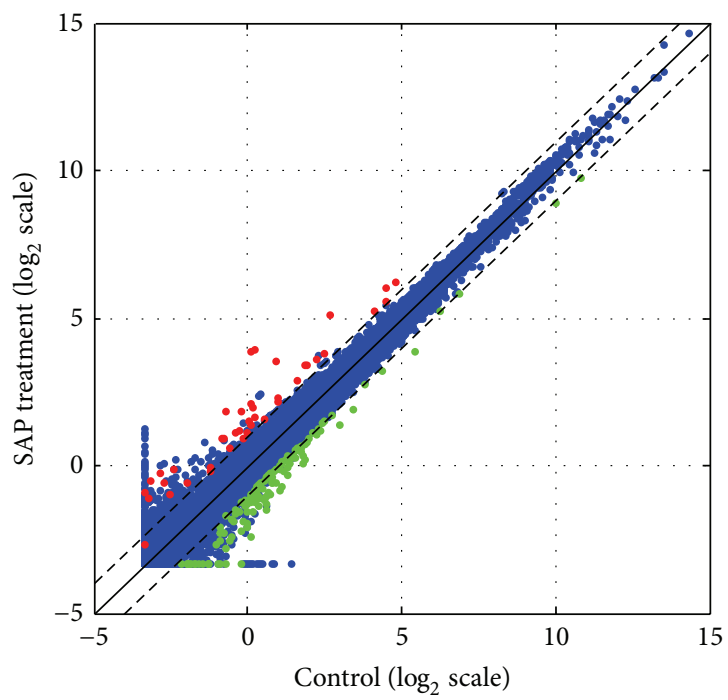

(c)

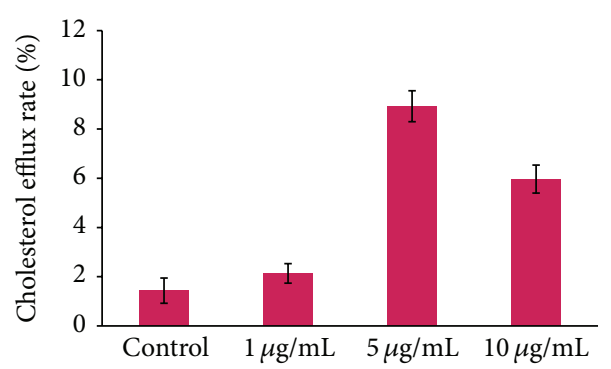

Control $\quad 1 \mu \mathrm{g} / \mathrm{mL} \quad 5 \mu \mathrm{g} / \mathrm{mL} \quad 10 \mu \mathrm{g} / \mathrm{mL}$

(b)

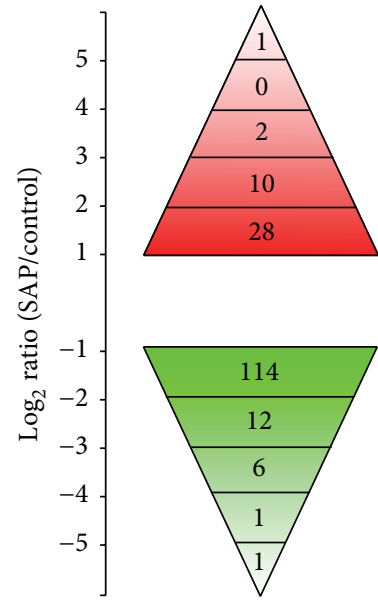

(d)

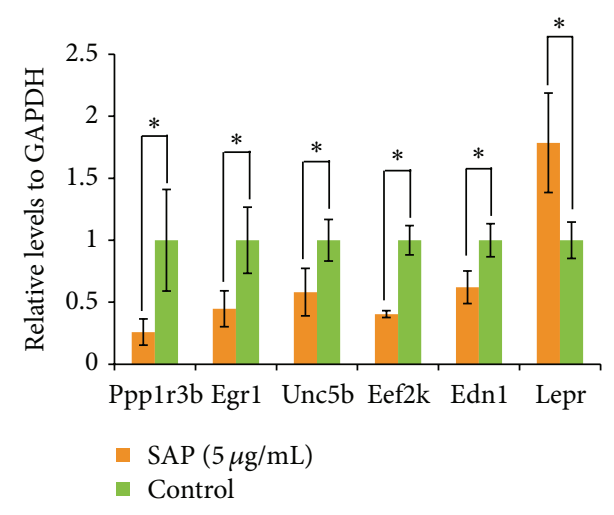

(e)

FIGURE 1: Systematic identification of genes that are altered in SAP treated cells compared to control cells. (a) SAP enhanced apoA1-mediated cholesterol efflux in a time-dependent manner in macrophages. (b) SAP enhanced apoAl-mediated cholesterol efflux in a dose-dependent manner in macrophages for $24 \mathrm{~h}$. (c) Scatter plot depicting the expression profiles of all genes. $\log _{2}$ transformed FPKM values from RNA sequencing were used in the scatter plot. We added 1 to FPKM value before $\log _{2}$ transformation to facilitate calculation. Nonchanged genes were shown in blue color while differently expressed genes (fold change $>2$ and FDR $<0.05$ ) were denoted in red or green. (d) Distribution of fold change of genes significantly different in SAP treated cells compared to control cells. (e) Validation of RNA sequencing data by quantitative RT-PCR. The GAPDH gene was used as the reference gene for normalization. The statistical significance was tested using unpaired 2-sample $t$-test. Values were plotted as means \pm standard error of the mean (SEM) of triplicate measurements. $n=3 .{ }^{*} p<0.05$.

were listed in Supplementary Table 1, available online at http://dx.doi.org/10.1155/2016/9380290.

\section{Results}

3.1. ApoAI-Mediated Cholesterol Efflux and Global Analysis of Gene Changes. SAP enhanced apoA1-mediated cholesterol efflux in a time- and dose-dependent manner in macrophages. After $10 \mu \mathrm{M}$ SAP treatment for $6 \mathrm{~h}$, the cholesterol efflux rate began to rise. The cholesterol efflux rate significantly increased after $24 \mathrm{~h}$ compared with $48 \mathrm{~h}$ (Figure 1(a)). Compared with control, after SAP treatment for $24 \mathrm{~h}, 5$ and $10 \mu \mathrm{M}$ SAP significantly increased the cholesterol efflux rate, but there was no significant difference with $1 \mu \mathrm{M}$ SAP treatment (Figure 1(b)). In order to investigate the molecular mechanism underlying SAP-mediated cholesterol efflux rate increase, transcriptome analysis was performed. For simplicity, cells were treated with $5 \mu \mathrm{M}$ for $24 \mathrm{~h}$ and then subjected to RNA sequencing analysis. We obtained $12,050,636$ reads from SAP treated cells and 11,651,186 reads from control cells, respectively. Among all reads obtained in this study, a total of $20,242,256(85.4 \%)$ reads were mappable to the mouse reference genome, of which $79.3 \%$ were mapped uniquely to only one location. Absolute gene expression levels were calculated in FPKM (fragments per kilobase of transcript per million mapped fragments) based on RefSeq gene models. We then compared the relative gene abundance in SAP treated cells and control cells. Differentially expressed genes were identified according to their fold changes $(>2)$ and false discovery rate $(\mathrm{FDR})$ adjusted $p$ values $(<0.05)$ (Figure $1(\mathrm{c})$ ). Compared to control, 134 genes were downregulated and 41 
genes were upregulated after SAP treatment (Supplementary Table 2). The range of fold changes was 41.01 2.00 and 54.14 2.01 for downregulated and upregulated genes, respectively (Figure 1(d)). In order to validate RNA-seq data, we randomly picked up 6 genes for qRT-PCR analysis. The expression trend of these genes measured by qRT-PCR was consistent with RNA sequencing data (Figure 1(e)), suggesting that our RNAseq data are of high quality.

3.2. Gene Ontology (GO) Analysis. All differentially expressed genes (134 downregulated and 41 upregulated) were functionally categorized based on gene ontology (GO) annotation terms using BiNGO software. Enrichment analysis revealed that a total of $9 \mathrm{GO}$ terms exhibited significance as overrepresented terms $(p<0.05)$. In the biological process category, 5 GO terms, namely, biosynthetic process, macromolecule metabolic process, nucleic acid metabolic process, response to stimulus, and cell death, were found to be significantly enriched. GO terms related to cell chromosome and nucleus were significantly enriched under the cellular component category. Enriched GO terms in the molecular function category were binding and nucleic acid binding. The hierarchical organization of these GO terms is shown in Figure 2(a), together with the significance of enrichment indicated by different colors. Strikingly, a total of 24 differentially expressed genes fell into the response to stimulus category. Included were 17 downregulated genes (Atr, Fancm, Tnfsf15, Thbd, Tlr13, F3, Acot11, Tlr3, Ahrr, Mt1, Edn1, Rif1, Mt2, Rrm2b, Lig4, Mlh3, and Kin) and 7 upregulated genes (Tnfsf10, H2-Ab1, Bc1, Ltb, Hspala, Gadd45g, and Hspalb) (Figure 2(b)).

3.3. Network Analysis of SAP-Regulated Genes. Network analysis can help understand the molecular and cellular interactions. It can be visualized to represent genes (nodes) and their relationships (edges). In the present study, we investigated functional interaction among SAP-regulated genes using the web-based network tool STRING (http://string-db.org/). The results from STRING were shown as Figure 3(a). The highly connected nodes, also known as hub genes, represent functionally important genes in the network. Connectivity analysis showed that Atr, Hspala, Hspalb, Mblac2, and Vrk1 were hubs of the network (Figure 3(b)). Additionally, 4 genes (Tnfsf15, Tlr3, Nlrp3, and Lepr), which have known roles in cholesterol efflux and atherosclerosis, were present in the network.

3.4. Altered Expression of Noncoding RNA Genes. Although the purpose of the present study was to measure polyadenylated mRNAs, it was possible that additional polyadenylated noncoding RNA genes might be present in our dataset. We examined all the 175 differentially expressed genes and discovered 19 noncoding RNA genes, including 11 long noncoding RNAs, 4 pseudogenes, 3 snRNA precursor/hosting RNAs, and 1 miRNA precursor/hosting RNA (Figure 4(a)). Among them, we validated the expression levels of 8 noncoding genes (Figure 4(b)). The expression trend of these genes measured by $\mathrm{qRT}-\mathrm{PCR}$ was consistent with RNA sequencing data
(Figure 4(c)). Strikingly, the miRNA hosting gene Mirl7hg is significantly downregulated which is resided on the plus strand of chromosome 14 (Figure $4(\mathrm{~d})$ ).

\section{Discussion}

Serum concentration of SAP in normal humans is about $30 \sim 40 \mu \mathrm{g} / \mathrm{mL}$. In healthy mice, normal SAP levels may be as low as $10 \mu \mathrm{g} / \mathrm{mL}$ in the C57BL strain and as high as $100 \mu \mathrm{g} / \mathrm{mL}$ (approximately $1 \mu \mathrm{M}$ ) in the DBA/2 strain [22]. In the present study, RAW264.7 mouse macrophages were used. Therefore, we chose $1 \mu \mathrm{M}$ to represent a physiological situation. Higher concentrations up to $10 \mu \mathrm{M}$ were used to mimic the acute phase reaction. We showed that SAP treatment induced macrophage cholesterol efflux with a time- and dose-dependent manner. The transcriptomic differences upon SAP treatment were determined by using RNA sequencing (RNA-seq). RNA-seq is an unbiased method which is not limited to detecting predesigned sequences [23]. In contrast to microarray, RNA-seq does not suffer from cross-hybridization [24]. Additionally, RNA-seq does not have any upper limit for quantification, making it a highly accurate tool for quantifying gene expression levels.

Through RNA sequencing, we identified a total of 175 differentially expressed genes, of which 134 genes were downregulated and 41 genes were upregulated after SAP treatment. Among these genes, 14 genes ( 6 protein-coding genes and 8 noncoding genes) were selected and validated by using qRT-PCR. In general, the expression trend of these genes measured by qRT-PCR was consistent with RNA sequencing, suggesting that our data were of high quality. Based on gene ontology analysis, a total of 9 GO terms exhibited significance as overrepresented terms: biosynthetic process, macromolecule metabolic process, nucleic acid metabolic process, response to stimulus, and cell death in the biological process category; cell chromosome and nucleus under the cellular component category; and binding and nucleic acid binding in the molecular function category. These results suggest that SAP treatment may have a wide effect on macrophages.

In our present study, we found that TNFSF15, TLR3, and NLRP3 were downregulated upon SAP treatment. Tumor necrosis factor superfamily member 15 (TNFSF15), also known as vascular endothelial growth inhibitor (VEGI) or TNF ligand related molecule 1A (TL1A), is a unique cytokine that functions as a modulator of vascular homeostasis and inflammation $[25,26]$. TNFSF15 is involved in numerous cellular processes including the suppression of neovascularization which is essential for tumor progression and spread $[26,27]$. TNFSF15 inhibits cholesterol efflux and suppresses the expression of three proteins, apoE, ABCA1, and ABCG1, both in vitro and in vivo $[28,29]$. Toll-like receptors (TLRs) are the most characterized innate immune receptors as well as pattern-recognition receptors (PRRs) [30, 31]. Scavenger receptors induced by TLR3 could regulate increased lipid uptake and activated TLR3 also increases TG accumulation in RAW cells [32, 33]. Indeed, stimulation of TLR3 or TLR4 by pathogen-derived ligands inhibits expression of 


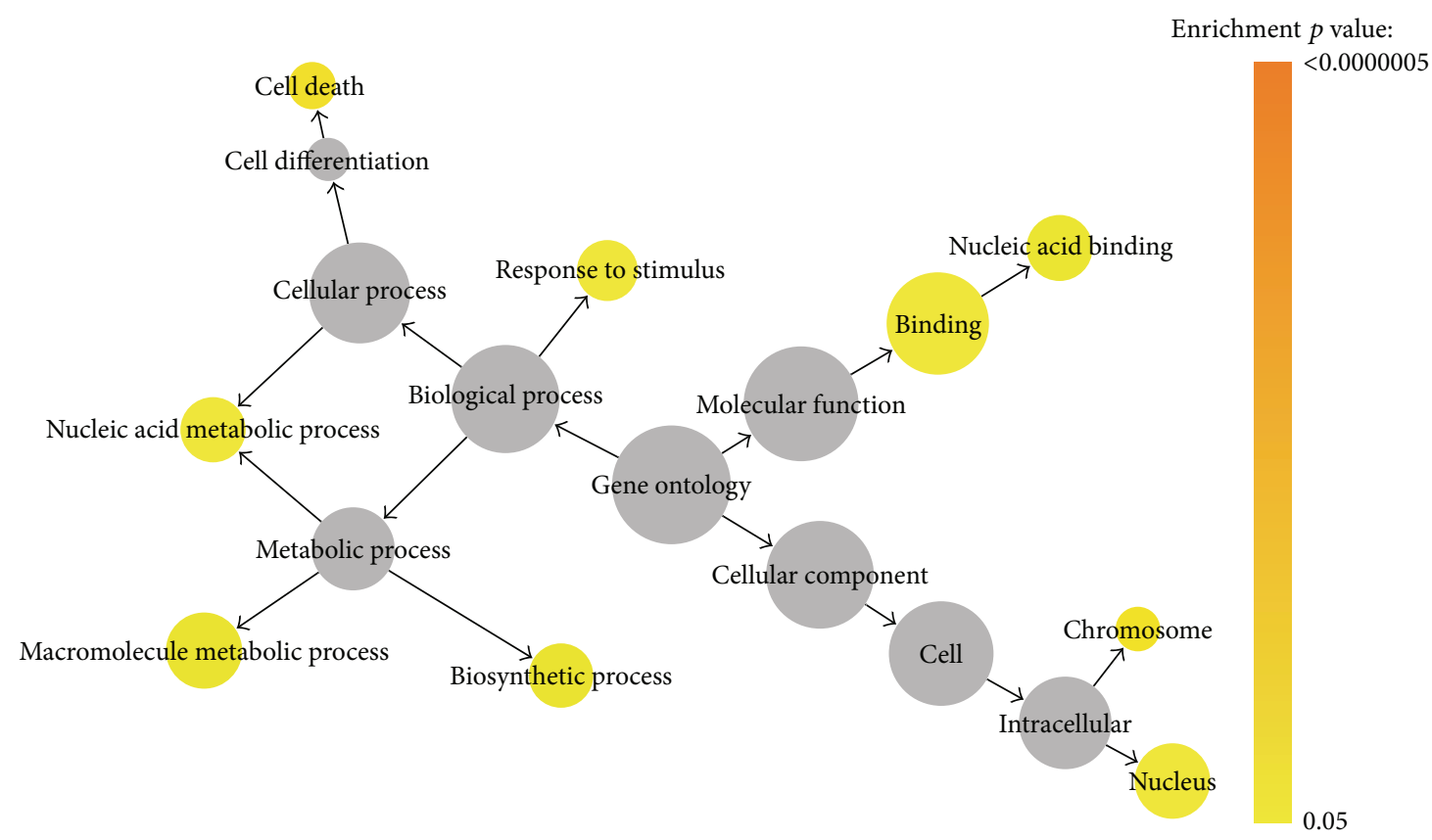

(a)

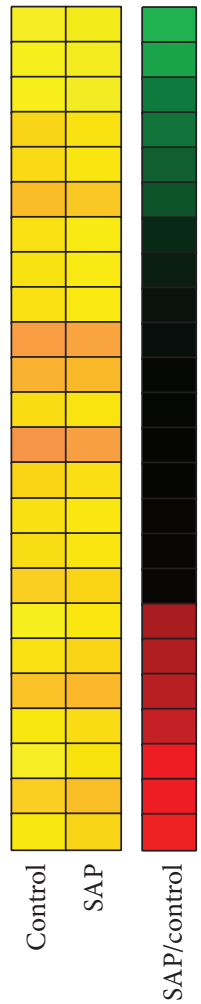

Ataxia telangiectasia and Rad3 related (Atr)

Fanconi anemia complementation group M (Fancm)

Tumor necrosis factor superfamily member 15 (Tnfsf15)

Thrombomodulin (Thbd)

Toll-like receptor 13 (Tlr13)

Coagulation factor III (F3)

Acyl-CoA thioesterase 11 (Acot11)

Toll-like receptor 3 (Tlr3)

Aryl-hydrocarbon receptor repressor (Ahrr)

Metallothionein 1 (Mt1)

Endothelin 1 (Edn1)

Rap1 interacting factor 1 homolog (Rif1)

Metallothionein 2 (Mt2)

Ribonucleotide reductase M2B (Rrm2b)

Ligase IV DNA ATP dependent (Lig4)

MutL homolog 3 (Mlh3)

Antigenic determinant of rec-A protein (Kin)

Tumor necrosis factor superfamily member 10 (Tnfsf10)

Histocompatibility 2 class II antigen A beta 1 (H2-Ab1)

Brain cytoplasmic RNA 1 (Bc1)

Lymphotoxin B (Ltb)

Heat shock protein 1A (Hspala)

Growth arrest and DNA-damage-inducible 45 gamma (Gadd45g)

Heat shock protein 1B (Hspalb)

苟

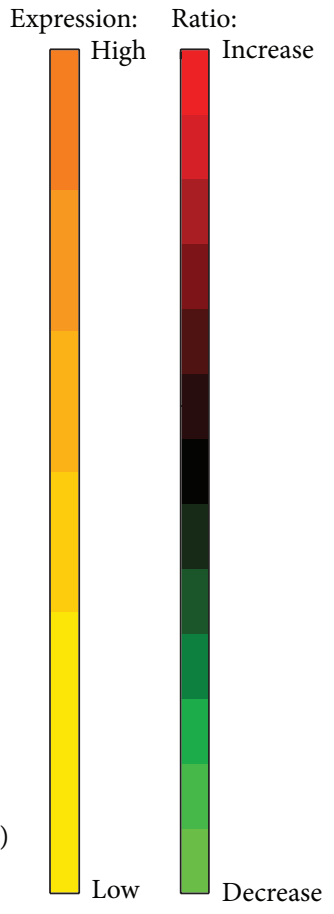

(b)

FIGURE 2: Functional clustering analysis of differentially expressed genes. (a) Differentially expressed genes were analyzed using BiNGO software. Significantly enriched GOslim categories were highlighted with different colors representing different levels of significance. The size of each circle is correlated to the number of genes. (b) Heat map of the 24 differentially expressed genes that fall into the response to stimulus category. In the heat map, the first and second columns correspond to the absolute gene expression levels (FPKM values) of SAP and control group, respectively. Values are color-coded, with yellow representing low levels and orange representing high levels. The third column of the heat map reports the relative expression levels. The values are the $\log _{2}$ ratio of SAP versus control. Red indicates increase and green indicates decrease. 


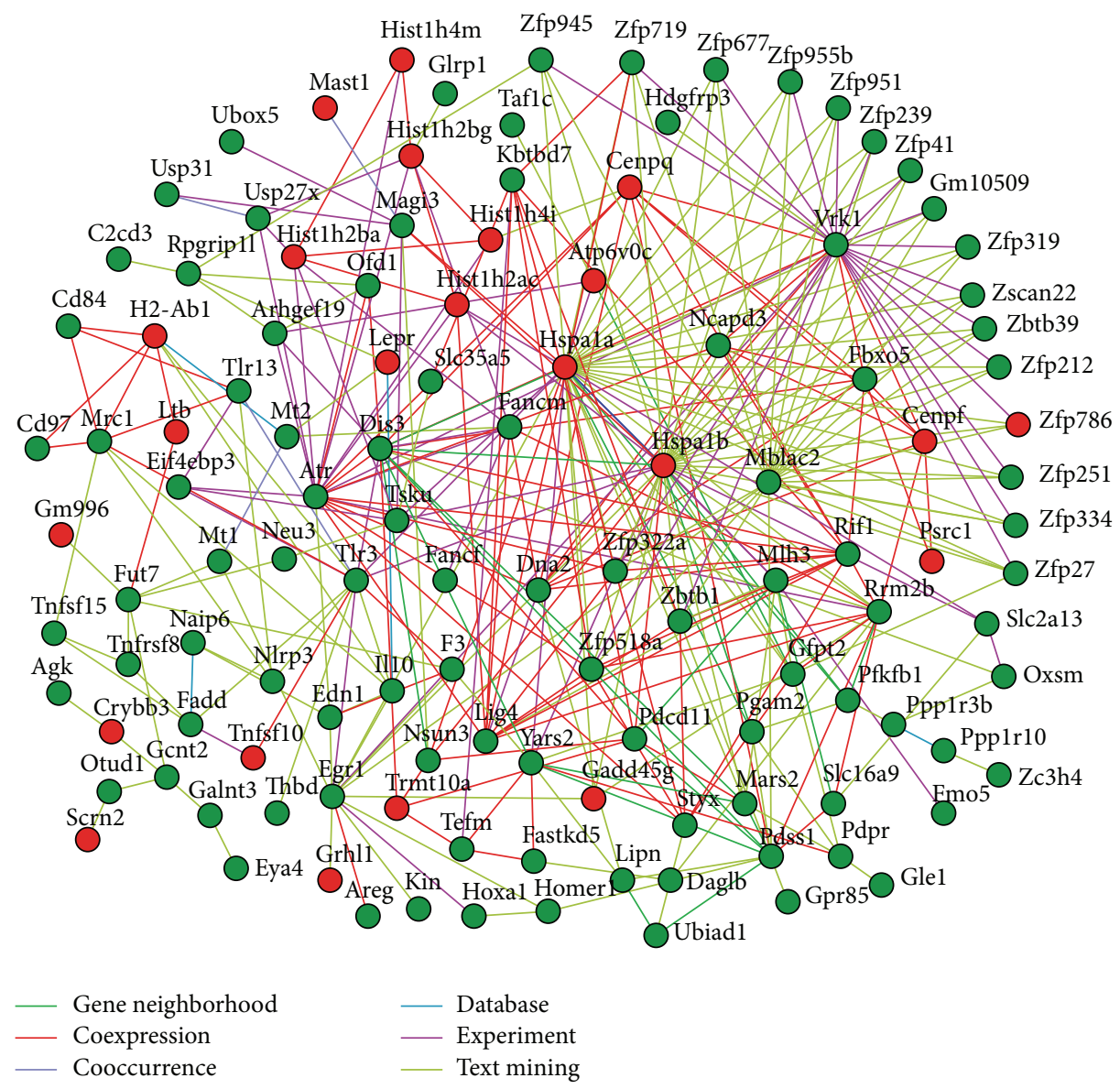

(a)

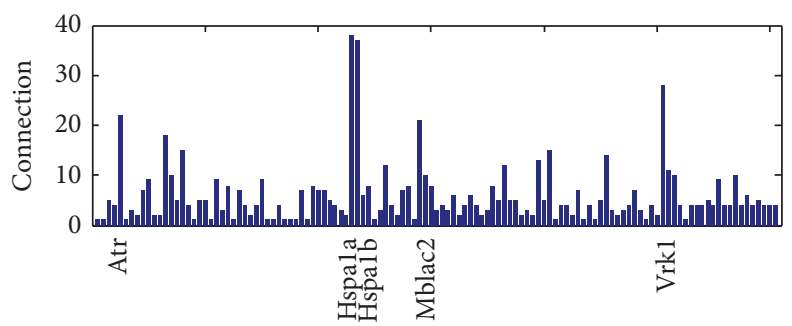

(b)

FIGURE 3: Network analysis of SAP-regulated genes. (a) The gene network generated by STRING. (b) Bar plot showing connection degrees for all genes.

LXR-dependent gene targets and macrophage cholesterol efflux via a MyD88-independent mechanism and involves IRF3 [34, 35]. The nucleotide-binding domain, leucinerich-containing family, pyrin domain-containing-3 (NLRP3) inflammasome has emerged as an important regulator of inflammation in metabolic disorders and atherosclerosis [36, 37]. Recent study also showed abnormal lipid deposition and lysosomal cholesterol accumulation are due to impaired intracellular lipid trafficking in macrophages upon Nlrp3 inflammasome activation by nonatherogenic stimulus ATP [38]. Moreover, mRNA levels of ABCA1 and ABCG1 were increased after the treatment of NLRP3i, suggesting that NLRP3 gene silencing could be a potentially therapeutic mean to increase macrophage cholesterol efflux for the prevention of atherosclerosis [39]. Therefore, elevated cholesterol efflux in macrophages by SAP may be mediated by the repression of TNFSF15, TLR3, and NLRP3.

Lepr, the leptin receptor, is expressed in many tissues including the cardiovascular system [40]. HDL-mediated cholesterol efflux was suppressed by leptin and expression of long form of Lepr was upregulated during monocytic differentiation into macrophages and sustained after differentiation [41]. In this study, we found that Lepr was upregulated upon SAP treatment, suggesting that SAP may also elevate cholesterol efflux via the upregulation of Lepr. 


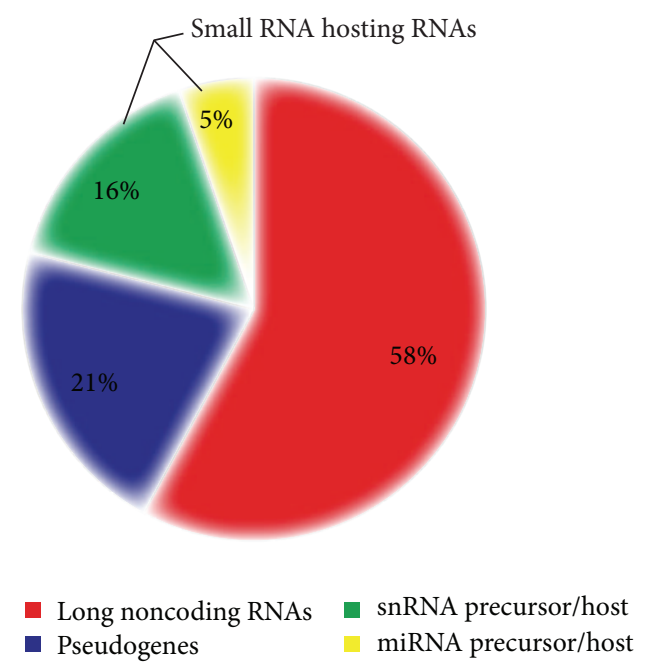

(a)

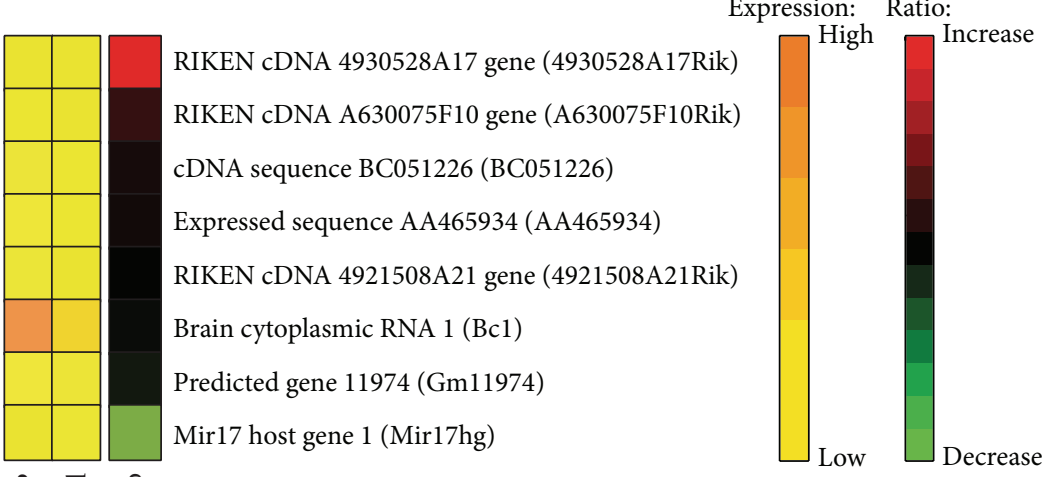

(b)

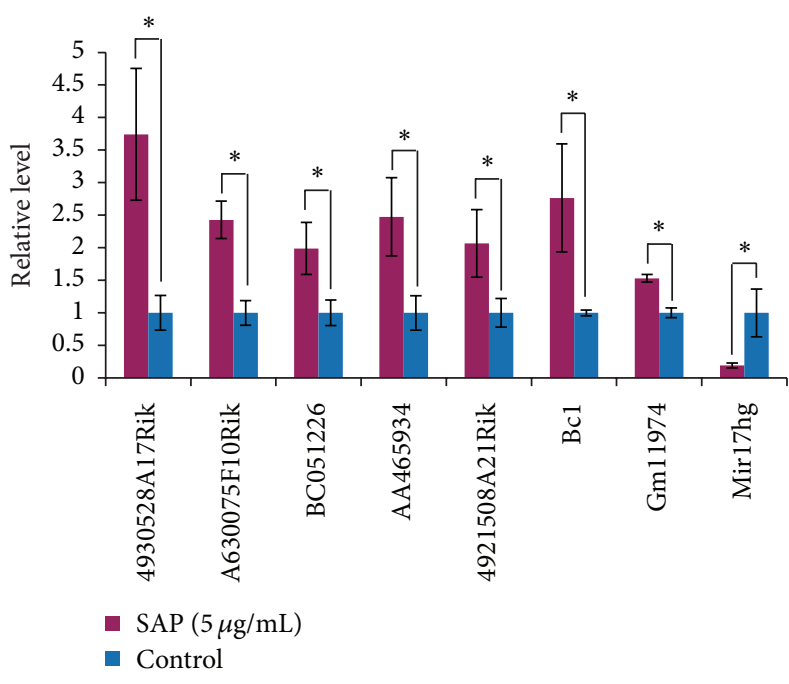

(c)

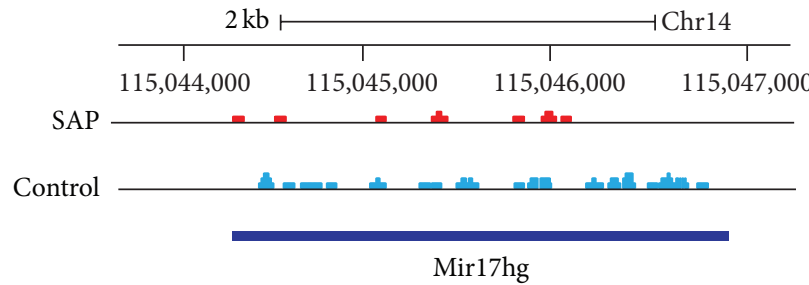

(d)

FIgURE 4: Changed expression of noncoding RNA genes. (a) Classification of noncoding RNA genes. Pie chart is displayed on the differently expressed 19 noncoding RNA genes. (b) Heat map of the 8 selected long noncoding RNA genes. The first and second columns correspond to the absolute gene expression levels (FPKM values) in SAP group and control group, respectively. The third column of the heat map reports the relative expression levels. Values are color-coded as indicated by the color bars. (c) Quantitative RT-PCR analysis of 8 selected lncRNAs. The GAPDH gene was used as the reference gene for normalization. Statistical significance was tested using unpaired 2-sample $t$-test. Values were plotted as means \pm standard error of the mean (SEM) of triplicate measurements. $n=3 .{ }^{*} p<0.05$. (d) RNA sequencing coverage plot of Mirl7hg lncRNA. Coverage plot is displayed on the reference genome (UCSC mm9). The upper panel represents expression in SAP group and the lower panel represents expression in control group. For both panels, numbers on $y$-axis refer to RNA sequencing read-depth at a given nucleotide position.

Although emerging evidence indicates long noncoding RNAs (lncRNAs) may have functional significance in development, physiology, and diseases [42], they remain unexplored on macrophage research. In this study, we identified 19 differentially expressed lncRNAs. Previously, the microRNA 17-92 cluster host gene (MIR17HG) has been shown to regulate expression of genes involved in breast cancer development and progression [43]. The functions of the other lncRNAs, including 6330549D23Rik, 4930528A17Rik, BC051226, AA465934, 4921508A21Rik, and Gm11974, have not been determined yet. Notably, SAP-induced genes such as Gadd45g are associated with cancer [44]. The idea that longterm use of SAP in patients might promote tumorigenesis deserves further investigation.

In conclusion, in the present study we found that SAP treatment increased cholesterol efflux rate. We analyzed global gene changes upon SAP treatment in RAW264.7 macrophages using RNA-seq. Our study may provide new insights into the molecular mechanisms underlying the functional role of SAP in macrophages. 


\section{Disclosure}

The sponsors had no role in the study design, data collection and analysis, our decision to publish, or the preparation of the paper.

\section{Competing Interests}

The authors declare that they have no competing interests.

\section{Acknowledgments}

This project was supported by the National Natural Science Foundation of China (81370380), the Natural Science Foundation of Guangdong Province of China (S2013010014739), and the Science and Technology Foundation of Guangdong Province of China (2012B091100155).

\section{References}

[1] D. Xi, T. Luo, H. Xiong et al., "SAP: structure, function, and its roles in immune-related diseases," International Journal of Cardiology, vol. 187, no. 1, pp. 20-26, 2015.

[2] J. Watanabe, V. Grijalva, S. Hama et al., "Hemoglobin and its scavenger protein haptoglobin associate with ApoA-1containing particles and influence the inflammatory properties and function of high density lipoprotein," The Journal of Biological Chemistry, vol. 284, no. 27, pp. 18292-18301, 2009.

[3] C. R. Stewart, A. A. Tseng, Y.-F. Mok et al., "Oxidation of lowdensity lipoproteins induces amyloid-like structures that are recognized by macrophages," Biochemistry, vol. 44, no. 25, pp. 9108-9116, 2005.

[4] C. Weber and H. Noels, "Atherosclerosis: current pathogenesis and therapeutic options," Nature Medicine, vol. 17, no. 11, pp. 1410-1422, 2011.

[5] C. Weber, A. Zernecke, and P. Libby, "The multifaceted contributions of leukocyte subsets to atherosclerosis: lessons from mouse models," Nature Reviews Immunology, vol. 8, no. 10, pp. 802-815, 2008.

[6] Z. Song, L. Cai, L. Guo, Y. Tsukamoto, C. Yutani, and X.-A. Li, "Accumulation and expression of serum amyloid $\mathrm{P}$ component in human atherosclerotic lesions," Atherosclerosis, vol. 211, no. 1, pp. 90-95, 2010.

[7] Y. Li, Q. Wu, Y. Deng et al., "D(-)-salicin inhibits the LPSinduced inflammation in RAW264.7 cells and mouse models," International Immunopharmacology, vol. 26, no. 2, pp. 286-294, 2015.

[8] Y.-C. Wang, H.-C. Hung, C.-W. Feng et al., "Dihydroaustrasulfone alcohol (WA-25) impedes macrophage foam cell formation by regulating the transforming growth factor- $\beta 1$ pathway," International Journal of Molecular Sciences, vol. 16, no. 5, pp. 10507-10525, 2015.

[9] N. Liu, C. Wu, L. Sun, J. Zheng, and P. Guo, "Sesamin enhances cholesterol efflux in RAW264.7 macrophages," Molecules, vol. 19, no. 6, pp. 7516-7527, 2014.

[10] T. Liu, C. Li, H. Sun et al., "Curcumin inhibits monocyte chemoattractant protein-1 expression and enhances cholesterol efflux by suppressing the c-Jun $\mathrm{N}$-terminal kinase pathway in macrophage," Inflammation Research, vol. 63, no. 10, pp. 841850, 2014.
[11] H. Sun, J. Shen, T. Liu et al., "Heat shock protein 65 promotes atherosclerosis through impairing the properties of high density lipoprotein," Atherosclerosis, vol. 237, no. 2, pp. 853-861, 2014.

[12] L. A. Murray, R. Rosada, A. P. Moreira et al., "Serum amyloid $\mathrm{P}$ therapeutically attenuates murine bleomycin-induced pulmonary fibrosis via its effects on macrophages," PLOS ONE, vol. 5, no. 3, Article ID e9683, 2010.

[13] S. B. Haudek, Y. Xia, P. Huebener et al., "Bone marrowderived fibroblast precursors mediate ischemic cardiomyopathy in mice," Proceedings of the National Academy of Sciences of the United States of America, vol. 103, no. 48, pp. 18284-18289, 2006.

[14] L. A. Murray, Q. Chen, M. S. Kramer et al., "TGF-beta driven lung fibrosis is macrophage dependent and blocked by Serum amyloid P," International Journal of Biochemistry and Cell Biology, vol. 43, no. 1, pp. 154-162, 2011.

[15] M. R. Dillingh, B. van den Blink, M. Moerland et al., "Recombinant human serum amyloid $\mathrm{P}$ in healthy volunteers and patients with pulmonary fibrosis," Pulmonary Pharmacology and Therapeutics, vol. 26, no. 6, pp. 672-676, 2013.

[16] D. Pilling, D. Roife, M. Wang et al., "Reduction of bleomycininduced pulmonary fibrosis by serum amyloid P," The Journal of Immunology, vol. 179, no. 6, pp. 4035-4044, 2007.

[17] Y. Tan, T. R. Liu, S. W. Hu et al., "Acute coronary syndrome remodels the protein cargo and functions of high-density lipoprotein subfractions," PLoS ONE, vol. 9, no. 4, Article ID e94264, 2014.

[18] S.-P. Zhao, J. Yang, J. Li, S.-Z. Dong, and Z.-H. Wu, "Effect of niacin on LXR $\alpha$ and PPAR $\gamma$ expression and HDL-induced cholesterol efflux in adipocytes of hypercholesterolemic rabbits," International Journal of Cardiology, vol. 124, no. 2, pp. 172178, 2008.

[19] C. Trapnell, L. Pachter, and S. L. Salzberg, “TopHat: discovering splice junctions with RNA-Seq," Bioinformatics, vol. 25, no. 9, pp. 1105-1111, 2009.

[20] C. Trapnell, B. A. Williams, G. Pertea et al., "Transcript assembly and quantification by RNA-Seq reveals unannotated transcripts and isoform switching during cell differentiation," Nature Biotechnology, vol. 28, no. 5, pp. 511-515, 2010.

[21] S. Maere, K. Heymans, and M. Kuiper, "BiNGO: a Cytoscape plugin to assess overrepresentation of gene ontology categories in biological networks," Bioinformatics, vol. 21, no. 16, pp. 34483449, 2005.

[22] M. B. Pepys, M. L. Baltz, F. C. de Beer et al., "Biology of serum amyloid P component," Annals of the New York Academy of Sciences, vol. 389, pp. 286-298, 1982.

[23] Z. Wang, M. Gerstein, and M. Snyder, "RNA-Seq: a revolutionary tool for transcriptomics," Nature Reviews Genetics, vol. 10, no. 1, pp. 57-63, 2009.

[24] A. Mortazavi, B. A. Williams, K. McCue, L. Schaeffer, and B. Wold, "Mapping and quantifying mammalian transcriptomes by RNA-Seq," Nature Methods, vol. 5, no. 7, pp. 621-628, 2008.

[25] S. Grimaldo, F. Tian, and L.-Y. Li, "Sensitization of endothelial cells to VEGI-induced apoptosis by inhibiting the NF- $\kappa \mathrm{B}$ pathway," Apoptosis, vol. 14, no. 6, pp. 788-795, 2009.

[26] Z. Zhang and L.-Y. Li, "TNFSF15 modulates neovascularization and inflammation," Cancer Microenvironment, vol. 5, no. 3, pp. 237-247, 2012.

[27] E. Zhang, X. Zhu, S. Han et al., "Increased expression of TNF ligand-related molecule $1 \mathrm{~A}$ and death receptor 3 in bladder tissues of patients with painful bladder syndrome/interstitial cystitis," Experimental and Therapeutic Medicine, vol. 5, no. 1, pp. 282-286, 2013. 
[28] A. R. Tall, L. Yvan-Charvet, N. Terasaka, T. Pagler, and N. Wang, "HDL, ABC transporters, and cholesterol efflux: implications for the treatment of atherosclerosis," Cell Metabolism, vol. 7, no. 5, pp. 365-375, 2008.

[29] J. E. McLaren, C. J. Calder, B. P. McSharry et al., "The TNFlike protein $1 \mathrm{~A}$-death receptor 3 pathway promotes macrophage foam cell formation in vitro," The Journal of Immunology, vol. 184, no. 10, pp. 5827-5834, 2010.

[30] J. E. Cole, A. T. Mitra, and C. Monaco, "Treating atherosclerosis: the potential of Toll-like receptors as therapeutic targets," Expert Review of Cardiovascular Therapy, vol. 8, no. 11, pp. 1619-1635, 2010.

[31] J. E. Cole, E. Georgiou, and C. Monaco, "The expression and functions of toll-like receptors in atherosclerosis," Mediators of Inflammation, vol. 2010, Article ID 393946, 18 pages, 2010.

[32] L. Shao, P. Zhang, Y. Zhang, Q. Lu, and A. Ma, "TLR3 and TLR4 as potential clinically biomarkers of cardiovascular risk in coronary artery disease (CAD) patients," Heart and vessels, vol. 29, no. 5, pp. 690-698, 2014.

[33] K. R. Feingold, J. K. Shigenaga, M. R. Kazemi et al., "Mechanisms of triglyceride accumulation in activated macrophages," Journal of Leukocyte Biology, vol. 92, no. 4, pp. 829-839, 2012.

[34] A. Castrillo, S. B. Joseph, S. A. Vaidya et al., "Crosstalk between LXR and Toll-like receptor signaling mediates bacterial and viral antagonism of cholesterol metabolism," Molecular Cell, vol. 12, no. 4, pp. 805-816, 2003.

[35] S. Chen, R. Sorrentino, K. Shimada et al., "Chlamydia pneumoniae-induced foam cell formation requires MyD88dependent and -independent signaling and is reciprocally modulated by liver X receptor activation," The Journal of Immunology, vol. 181, no. 10, pp. 7186-7193, 2008.

[36] P. Duewell, H. Kono, K. J. Rayner et al., "NLRP3 inflammasomes are required for atherogenesis and activated by cholesterol crystals," Nature, vol. 464, pp. 1357-1361, 2010.

[37] B. Vandanmagsar, Y.-H. Youm, A. Ravussin et al., “The NLRP3 inflammasome instigates obesity-induced inflammation and insulin resistance," Nature Medicine, vol. 17, no. 2, pp. 179-189, 2011.

[38] X. Li, Y. Zhang, M. Xia, E. Gulbins, K. M. Boini, and P.-L. $\mathrm{Li}$, "Activation of Nlrp3 inflammasomes enhances macrophage lipid-deposition and migration: implication of a novel role of inflammasome in atherogenesis," PLoS ONE, vol. 9, no. 1, Article ID e87552, 2014.

[39] F. Zheng, S. Xing, Z. Gong, W. Mu, and Q. Xing, "Silence of NLRP3 suppresses atherosclerosis and stabilizes plaques in apolipoprotein E-deficient mice," Mediators of Inflammation, vol. 2014, Article ID 507208, 8 pages, 2014.

[40] J. Beltowski, "Leptin and atherosclerosis," Atherosclerosis, vol. 189, no. 1, pp. 47-60, 2006.

[41] S. Hongo, T. Watanabe, S. Arita et al., "Leptin modulates ACAT1 expression and cholesterol efflux from human macrophages," American Journal of Physiology-Endocrinology and Metabolism, vol. 297, no. 2, pp. E474-E482, 2009.

[42] A. Fatica and I. Bozzoni, "Long non-coding RNAs: new players in cell differentiation and development," Nature Reviews Genetics, vol. 15, no. 1, pp. 7-21, 2014.

[43] D. Chacon-Cortes, R. A. Smith, R. A. Lea, P. H. Youl, and L. R. Griffiths, "Association of microRNA 17-92 cluster host gene (MIR17HG) polymorphisms with breast cancer," Tumor Biology, vol. 36, no. 7, pp. 5369-5376, 2015.
[44] B. Hoffman and D. A. Liebermann, "Gadd45 in modulation of solid tumors and leukemia," Advances in Experimental Medicine and Biology, vol. 793, pp. 21-33, 2013. 

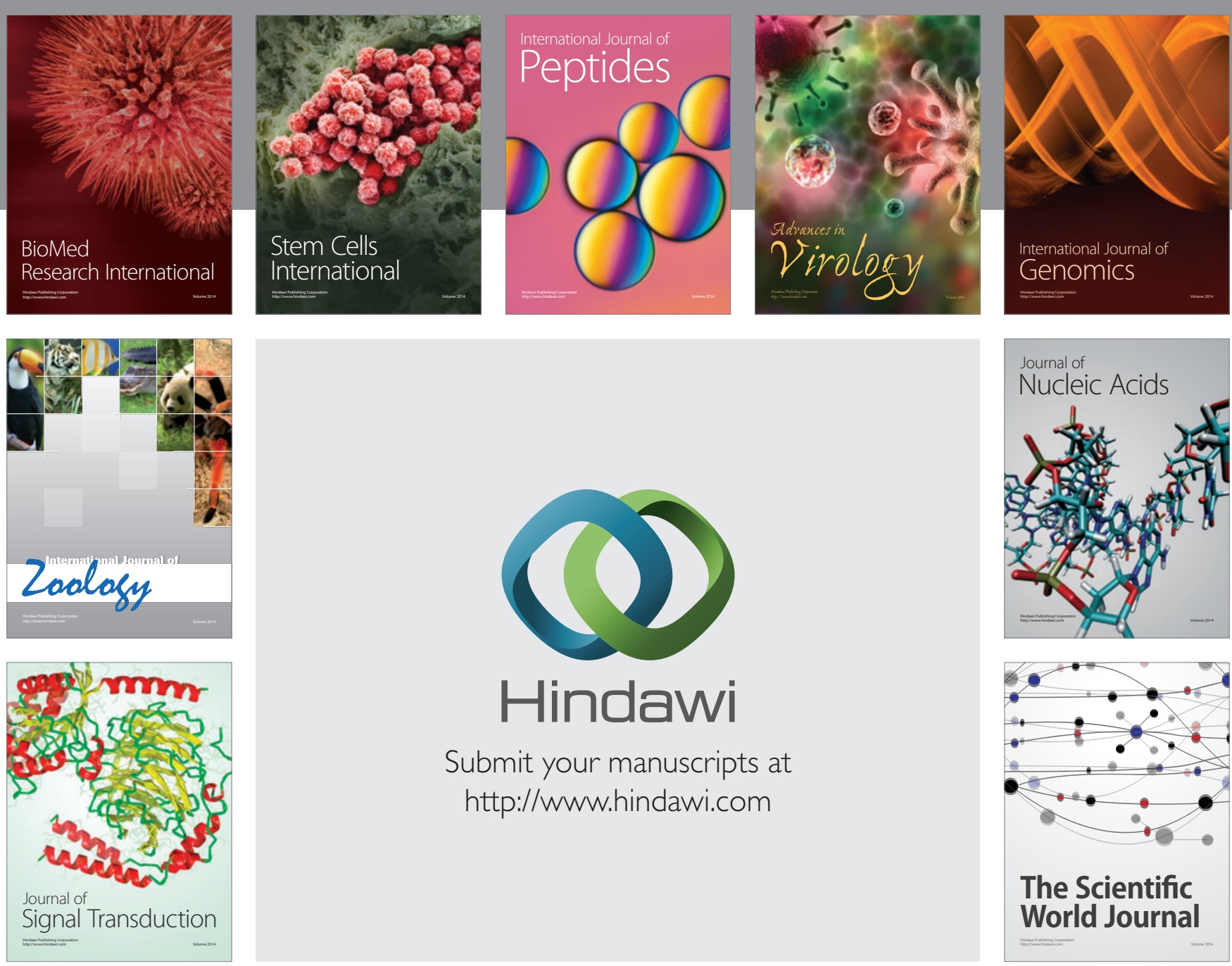

Submit your manuscripts at

http://www.hindawi.com
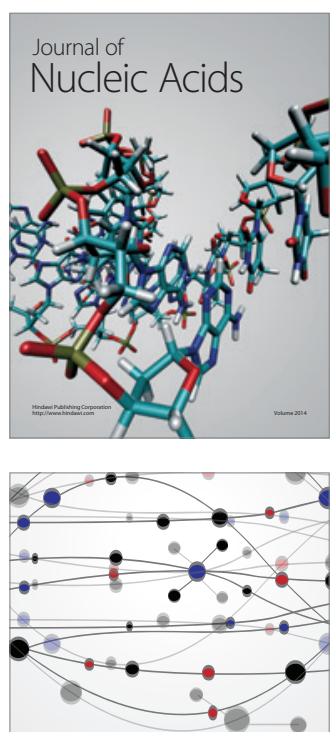

The Scientific World Journal
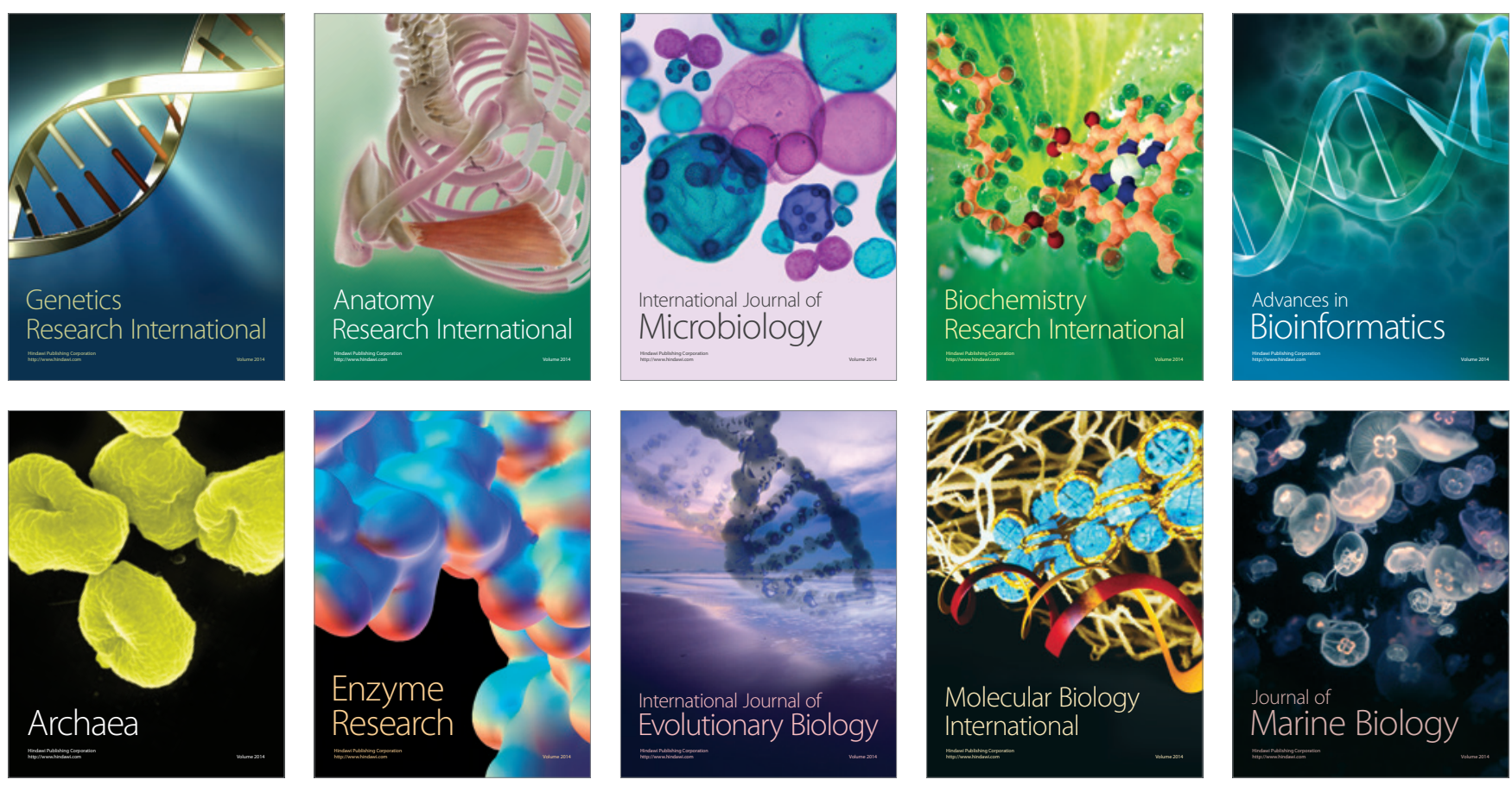